\title{
quimb: A python package for quantum information and many-body calculations
}

\section{DOI: $10.21105 /$ joss.00819}

\section{Software}

- Review ¿t

- Repository u

- Archive ${ }^{\top}$

Submitted: 14 June 2018

Published: 04 September 2018

\section{License}

Authors of papers retain copyright and release the work under a Creative Commons Attribution 4.0 International License (CC-BY).

\section{Johnnie Gray ${ }^{1}$}

1 University College London, London, UK

\section{Summary}

The language of quantum physics is essentially linear algebra, making it easy to begin simulating using standard numerical routines. However, the amount of classical resources required to simulate a quantum system scales exponenially with its size. This imposes, in the generic case, dramatic limits on the sizes reachable and requires that great care is taken in order to maximise performance. Nonetheless, and in part due to this difficulty, there is much to be learnt from simulating many-body quantum systems. One useful set of tools in this case is quantum information inspired quantities such as entanglement meausures. Entanglement is also the key quantity when formulating tensor networks, an efficient representation of certain many-body quantum states. quimb is a pure python library that covers all these areas, with an emphasis on being interactive and easy to use without sacrificing performance.

The main quimb module utilizes numpy (Guide to numpy, 2015) and scipy (Jones, Oliphant, Peterson, \& others, 2001-2001--) sparse matrices to represent quantum states and operators. Amongst other things there are tools to: (i) construct states and operators in composite tensor spaces; (ii) generate various special or random states and operators such as Hamiltonians; (iii) perform and compute many operations and quantities on these states; and (iv) efficiently evolve states with a variety of methods. Much of this core functionality is accelerated with numba or numexpr. Additionally, quimb has an optional slepc4py (Dalcin, Paz, Kler, \& Cosimo, 2011; Hernandez, Roman, \& Vidal, 2005) interface for various linear algebra routines, such as eigen-decomposition. These are accessed through unified functions so that one can easily switch between slepc4py, scipy and other backends. When required, quimb handles spawning local mpi4py (Dalcín, Paz, \& Storti, 2005) workers automatically, but there also tools for explicitly running under MPI, for example on a cluster. The following snippet illustrates some basic usage:

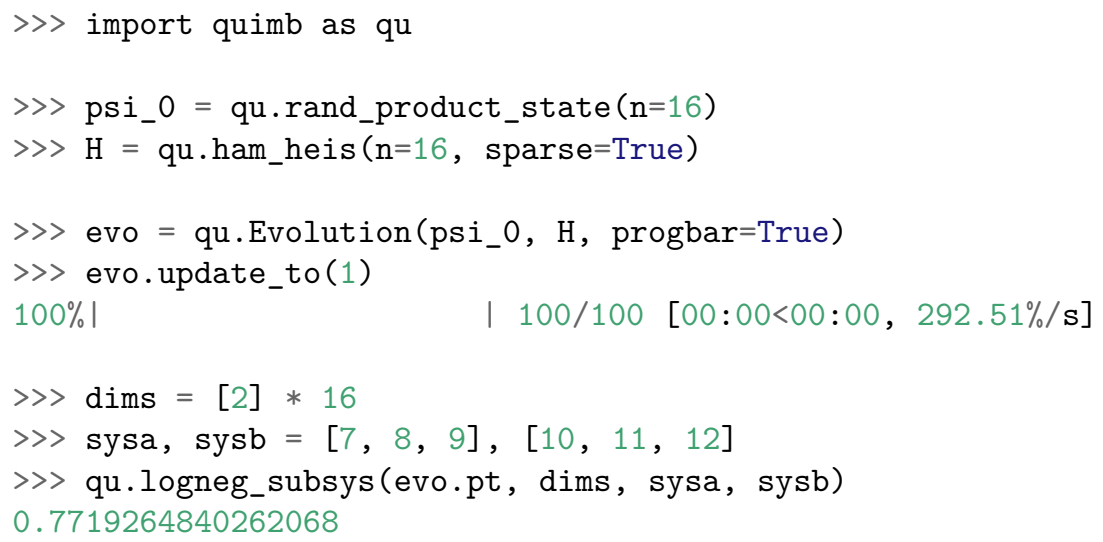

The submodule quimb.tensor has a more speciliazed set of tools that focus on tensor networks (Bridgeman \& Chubb, 2017) - one of the key recent advancements in many-body 


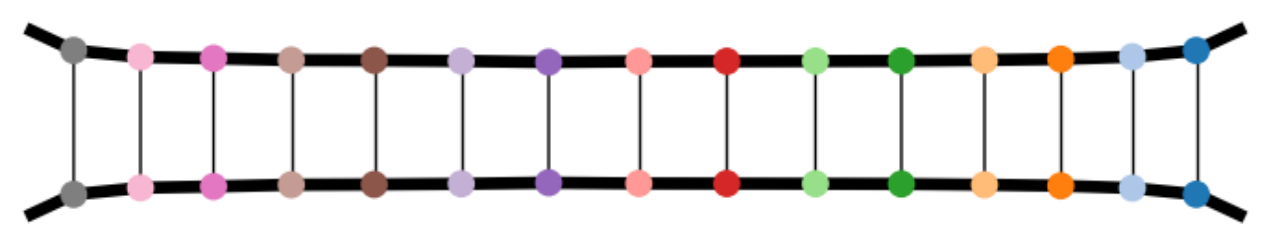

Figure 1: Example output of the tensor network graph plotter.

quantum theory. General highlights of quimb.tensor include: (i) an efficient, geometry free representation of arbitrary tensor networks; (ii) automatic contraction of 100s-1000s of tensors using opt_einsum (D. G. Smith \& Gray, 2018), including on the GPU; (iii) the ability to plot any tensor network, color-coded, with bond-sizes represented; and (iv) the ability to treat any network as a LinearOperator, allowing many iterative decompositions such as those in scipy. Based on these, fast versions of $1 \mathrm{D}$ tensor network algorithms such as DMRG and TEBD are implemented, as well as tools for efficiently dealing with periodic boundary conditions. The following snippet illustrates some usage of quimb.tensor:

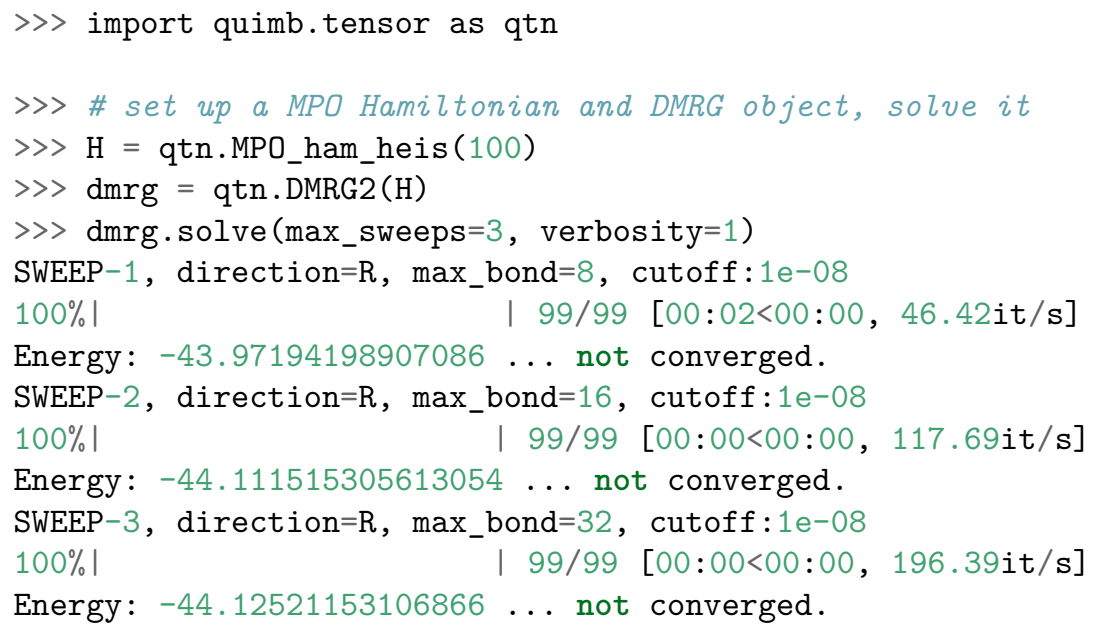

Overall, quimb aims to be an accessible but capable approach to simulating quantum many-body problems, and has been used already in a number of publications(Gray, Banchi, Bayat, \& Bose, 2017; Gray, Bayat, Puddy, Smith, \& Bose, 2016; Gray, Bose, \& Bayat, 2018). The full documentation can be found online at http://quimb.readthedocs. io/en/latest/. 


\section{Acknowledgements}

JG acknowledges funding from the EPSRC Centre for Doctoral Training in Delivering Quantum Technologies at UCL.

\section{References}

Bridgeman, J. C., \& Chubb, C. T. (2017). Hand-waving and interpretive dance: An introductory course on tensor networks. Journal of Physics A: Mathematical and Theoretical, 50(22), 223001. doi:10.1088/1751-8121/aa6dc3

Dalcin, L. D., Paz, R. R., Kler, P. A., \& Cosimo, A. (2011). Parallel distributed computing using python. Advances in Water Resources, 34(9), 1124-1139. doi:10.1016/j.advwatres.2011.04.013

Dalcín, L., Paz, R., \& Storti, M. (2005). MPI for python. Journal of Parallel and Distributed Computing, 65(9), 1108-1115. doi:10.1016/j.jpdc.2005.03.010

Gray, J., Banchi, L., Bayat, A., \& Bose, S. (2017). Measuring entanglement negativity. arXiv preprint arXiv:1709.04923.

Gray, J., Bayat, A., Puddy, R. K., Smith, C. G., \& Bose, S. (2016). Unravelling quantum dot array simulators via singlet-triplet measurements. Physical Review B, 94(19), 195136. doi:10.1103/PhysRevB.94.195136

Gray, J., Bose, S., \& Bayat, A. (2018). Many-body localization transition: Schmidt gap, entanglement length, and scaling. Physical Review B, 97(20), 201105. doi:10.1103/PhysRevB.97.201105

Guide to numpy. (2015). (2nd ed.). USA: CreateSpace Independent Publishing Platform.

Hernandez, V., Roman, J. E., \& Vidal, V. (2005). SLEPc: A scalable and flexible toolkit for the solution of eigenvalue problems. ACM Transactions on Mathematical Software (TOMS), 31(3), 351-362. doi:10.1145/1089014.1089019

Jones, E., Oliphant, T., Peterson, P., \& others. (2001-2001--). SciPy: Open source scientific tools for Python. Retrieved from http://www.scipy.org/

Smith, D. G., \& Gray, J. (2018, june).Journal of Open Source Software. doi:10.21105/joss.00753 